\title{
Multiple Port Site Metastases After Laparoscopic Gastrectomy for Cancer
}

\author{
Amani Jambhekar, MD, Josue Chery, MD, Krystyna Kabata, PA, Piotr Gorecki, MD \\ Department of Surgery, New York Methodist Hospital, Brooklyn, New York, USA (all authors)
}

\begin{abstract}
Introduction: Port site metastases are known phenomena associated with laparoscopic resection of intra-abdominal malignancies, but have not been well documented for gastric cancer. We report a case of port site metastases after laparoscopic subtotal gastrectomy for advanced gastric adenocarcinoma.

Case Description: A 71-year-old woman with a history of hypertension and diabetes mellitus presented with melena, weight loss, and signs of gastric outlet obstruction. Preoperative workup demonstrated a T3N1M0 mass extending along the lesser curvature of the stomach with biopsy confirming adenocarcinoma. The patient underwent an uneventful laparoscopic subtotal gastrectomy with D2 lymphadenectomy followed by adjuvant chemotherapy. Thirteen months after surgery, the patient presented with palpable subcutaneous nodules at two of the port sites. computed tomographic (CT) scan confirmed the isolated nodules without distant metastases and fine-needle aspirations confirmed gastric adenocarcinoma. The patient was treated with another cycle of chemotherapy. A post treatment proton emission tomography (PET) scan did not show any other lesions, and the patient was scheduled for resection. During surgery the left upper quadrant mass was found to infiltrate the left colon and an additional mass was found at the prior umbilical port. Pathology was consistent with gastric adenocarcinoma for all the lesions. An excisional biopsy of the right upper quadrant lesion was completed.
\end{abstract}

Conclusion: Given the rarity of port site metastases after gastric adenocarcinoma, there is no conclusive literature regarding the management. Repeat chemotherapy followed by resection, if feasible, appears to be the most reasonable therapeutic intervention if there is no evidence of distant metastases.

Key Words: Chemotherapy, Gastric adenocarcinoma, Laparoscopic gastrectomy, Port site metastasis

Citation Jambhekar A, Chery J, Kabata K, Gorecki P. Multiple port site metastases after laparoscopic gastrectomy for cancer. CRSLS e2016.00005. DOI: 10.4293/CRSLS.2016.00005.

Copyright $(2016$ by SLS, Society of Laparoendoscopic Surgeons. This is an open-access article distributed under the terms of the Creative Commons Attribution-Noncommercial-ShareAlike 3.0 Unported license, which permits unrestricted noncommercial use, distribution, and reproduction in any medium, provided the original author and source are credited.

Disclosure: None reported.

Address correspondence to: Amani Jambhekar, MD, Department of Surgery, New York Methodist Hospital, 506 Sixth Street, Brooklyn, NY 11215. E-mail: amani.jambhekar@gmail.com

\section{INTRODUCTION}

The overall reported incidence for port site metastasis is $0.71 \%$, but a lower incidence of $0.33 \%$ is observed in patients with isolated trocar recurrences without evidence of other metastatic disease. ${ }^{1}$ This complication was first described in the literature in $1985,{ }^{2}$ and various reports emerged in the 1990s detailing port site recurrences in patients with colorectal, gallbladder, and ovarian malignances. In comparison, there are few data about the incidence of recurrent disease at a laparoscopic port site after staging and curative resection for gastric malignancy. Sev- eral theories have been proposed regarding the pathophysiology. We describe a port site recurrence after curative resection of a gastric adenocarcinoma. We discuss our management in conjunction with published literature.

\section{CASE DESCRIPTION}

A 71-year-old woman presented with anemia, black tarry stools, generalized weakness, postprandial nonbilious emesis, and a reported 15-20 lb weight loss over two months. The patient was cachectic with decreased skin turgor. Her preoperative laboratory evaluation revealed a 
hemoglobin level of $5.1 \mathrm{~g} / \mathrm{dL}$ and an albumin of $2.1 \mathrm{~g} / \mathrm{dL}$. The patient underwent an upper endoscopy which demonstrated a large mass extending from the gastric body to the pylorus. Pathology was consistent with adenocarcinoma, tubular type, moderately differentiated with ulceration. Further staging with endoscopic ultrasound and a computed tomography (CT) scan of the chest, abdomen, and pelvis demonstrated that the mass was T3N1M0 according to the American Joint Committee on Cancer staging system. A decision at tumor board was made for surgical resection followed by adjuvant chemotherapy. She underwent an uneventful laparoscopic subtotal gastrectomy with D2 lymphadenectomy. Her postoperative course was uneventful, and she was discharged after nine days. The pathology was consistent with a $9.5 \times 8.5-\mathrm{cm}$ tubular type gastric carcinoma, with clear margins. Six of 40 lymph nodes were positive for metastases. She then underwent adjuvant chemotherapy with five cycles of fluorouracil with leucovorin for four months. In the midst of her chemotherapy course at five months after surgery, the patient presented to the emergency department with abdominal pain and underwent CT of the abdomen and pelvis, which was negative for recurrent disease. During a routine follow-up visit thirteen months after surgery, subcutaneous nodules were palpated at two of the working port sites. She underwent fine-needle aspirations, which confirmed gastric adenocarcinoma. The paramedian incision from which the specimen had been extracted was well healed without any evidence of local recurrence. A subsequent CT confirmed the isolated nodules without distant metastases (Figure 1).

The patient then underwent an additional nine week course of chemotherapy with docetaxel. During this time, she was admitted for intractable epistaxis and symptomatic anemia. After the nine week treatment, the patient presented with decreased appetite and increased weight loss that was attributed to toxicity from her chemotherapy. Over the next several months the patient had visible enlargement of her subcutaneous nodules. A proton emission tomography (PET) scan did not show any other metastases, and resection was planned approximately four months after her last cycle of chemotherapy and 21 months after her initial surgery. She was brought to the operating room for exploratory laparotomy with an initial plan to resect both the right and left upper quadrant nodules (Figure 2).

During surgery, at the time of the excision of the right upper quadrant mass, the left upper quadrant mass was found to infiltrate the left colon. A previously unseen metastatic deposit was also noted at the umbilicus. There-

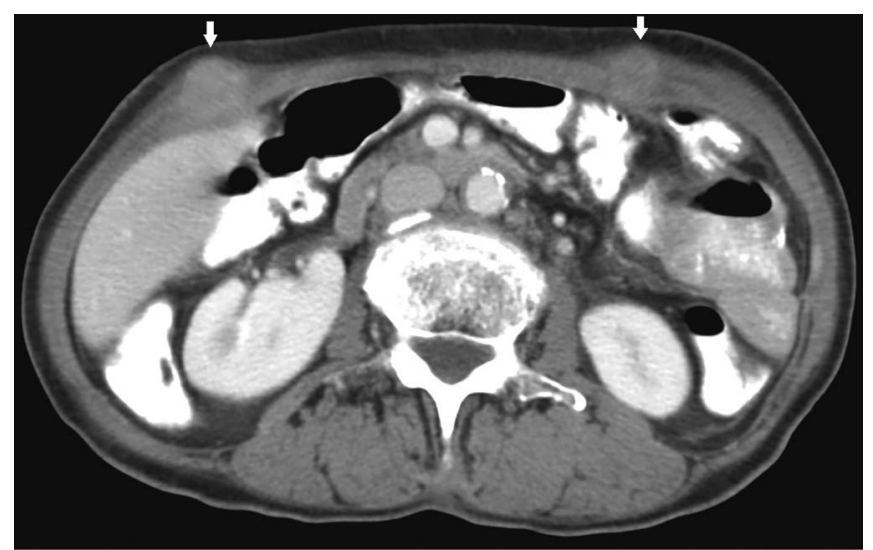

Figure 1. CT depicting metastatic deposits at bilateral upper quadrants.

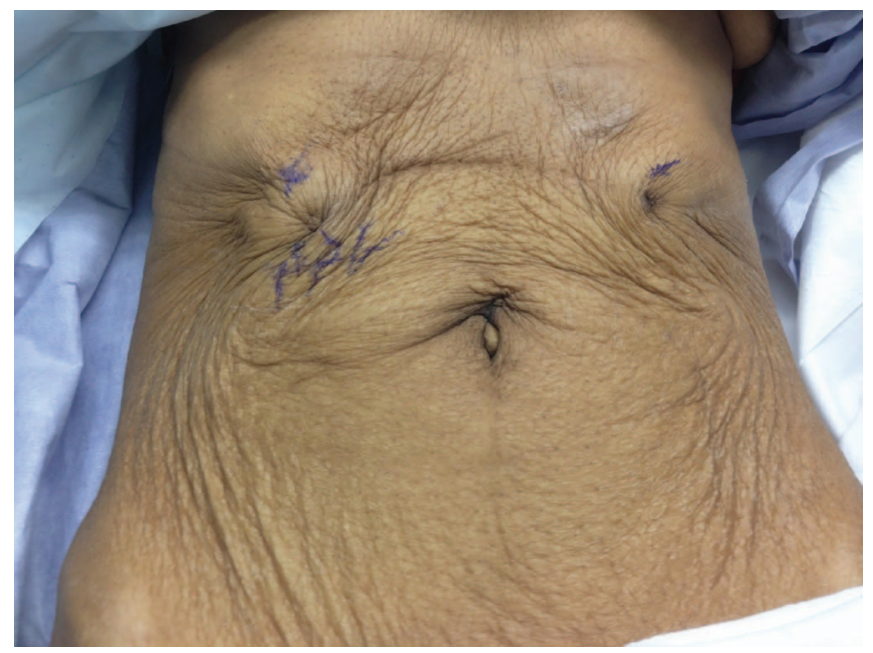

Figure 2. Intraoperative photograph depicting subcutaneous nodules.

fore, the decision was made not to proceed with extensive multiple resections of the abdominal wall which would require major and complex reconstruction with likely no additional survival benefit to the patient. The excision of the right upper quadrant nodule, however, was completed as planned, because it was technically simple as an excisional biopsy. A biopsy of the umbilical lesion was performed during surgery, which revealed adenocarcinoma. Peritoneal fluid on postoperative cytology was negative for malignancy. Notably, the paramedian incision through which the original specimen had been extracted was grossly normal. The postoperative course was uneventful, and the patient was discharged on postoperative day 1. She died seven weeks later of progressive disease, two years after her initial surgery. 


\section{DISCUSSION}

Port site metastases after resection for gastric adenocarcinoma are rarely reported. Pearlstone et $a^{3}$ included 1 patient in their study who had 1 port site metastasis after an Ivor Lewis esophagectomy for cancer of the gastroesophageal junction. The port site lesion was resected, and the patient remained disease free for 16 months after surgery. Schaeff et $\mathrm{al}^{4}$ included six cases of port site metastases after laparoscopy for gastric cancer in their 164 total cases of port site metastases. Five of the six cases underwent staging laparoscopy that subsequently revealed metastatic disease. To date, there have only been three detailed case reports of port site metastases after laparoscopic surgery for gastric cancer (Table 1) ${ }^{5-7}$ Two of the patients underwent surgery for diagnostic or palliative purposes.

Sakurai et $\mathrm{al}^{6}$ proposed that port site metastases are a result of a multifactorial process. Their patient presented with a T1b tumor and underwent laparoscopic-assisted distal gastrectomy. The authors hypothesize that dissemination from the primary tumor was unlikely and proposed that cancer cells in micrometastases of the lymphoid tissue may have disseminated to the port site during the lymph node dissection. According to Yasuda et $\mathrm{al}^{8}$ micrometastases in histologically negative lymph nodes were found in 20 of 64 patients (20\%). The presence of micrometastases was associated with significantly decreased 5-year survival. Direct wound contamination is also theorized to be a cause of port site metastases, but $40 \%$ of patients develop recurrences at sites other than the extraction site, which further complicates this theory.? Wound contamination is unlikely in our case, as a specimen bag was used in the original operation and the extraction site was free of metastatic disease, as confirmed by CT, PET, and intraoperative examination.

Local tissue trauma may contribute to both wound contamination and depression of local immune function. ${ }^{10}$ Jones et $\mathrm{al}^{11}$ established that tumor preferentially seeds to traumatized tissue. High cellular proliferation after tissue trauma predisposes to growth of neoplastic cells. ${ }^{12} \mathrm{Clot}$ formation at port sites also provides a fibrin-rich substrate and changes in macrophage and cytokine function provide a suitable environment for tumor growth. ${ }^{10}$ Tumor emboli may also become implanted in the wound secondary to changes in splanchnic circulation. ${ }^{13}$ Carbon dioxide may additionally depress immune function by impairing macrophages and cytokines via an inflammatory reaction of the peritoneal surface. ${ }^{10}$ Surgical technique is also an established theory behind port site metastases, as increased tumor handling causes intraoperative spillage. ${ }^{10}$ Repeated removal of contaminated instruments through the port sites or increased manipulation of trocars also may enhance tumor cell inoculation. ${ }^{1}$ Indirect contamination of the trocars is also possible via the "chimney effect" which suggests that tumor cells are preferentially translocated to port sites because of microleakages around the trocars. ${ }^{1}$

Minimal handling of the tumor, the use of a specimen bag for extraction, and the use of a standardized surgical approach that adheres to strict oncologic principles have all been suggested as methods by which to prevent port site recurrences. ${ }^{1,10}$ Prevention of gas leaks, avoiding sudden loss of pneumoperitoneum and closure of the peritoneum have also been described to ad preventive port site metastases. ${ }^{12,14}$ Surgeons should also be aware of the possibility of micrometastases to the lymph nodes, even when histologically negative, ${ }^{8}$ and therefore adjuvant therapy should be considered.

Despite the potential risk of port site metastases, laparoscopy remains a safe option. Deogracias et $\mathrm{al}^{15}$ used diagnostic laparoscopy as a screening method to determine the presence of absence of peritoneal metastases. Of the 41 patients studied, 59.5\% had stage II or higher gastric adenocarcinoma, and 88\% went forward with laparotomy and open gastrectomy. None of the patients developed port site or laparotomy incision metastases.

Table 1.

Patient Characteristics

\begin{tabular}{lllll}
\hline Year & Patient Age (y) & Procedure & Time to Metastases & Author(s) \\
\hline 1990 & 80 & Diagnostic laparoscopy & 7 days & ${\mathrm{Cava} \mathrm{et} \mathrm{al}^{7}}$ \\
2007 & 53 & Diagnostic laparoscopy and palliative gastrojejunostomy & 8 weeks & Rangarajan et al $^{2}$ \\
2013 & 57 & Laparoscopic-assisted distal gastrectomy & 18 months & Sakurai et al $^{6}$ \\
\hline All patients were men. & & & \\
\hline \hline
\end{tabular}




\section{CONCLUSION}

Multiple theories have been put forward regarding the pathogenesis of port site metastases. Few studies have been conducted to search for the cause or describing the incidence of port site metastases after laparoscopic resection for gastric adenocarcinoma. Based on the literature review, adjuvant chemotherapy should be considered, followed by local resection if possible, provided that there is no evidence of metastatic disease. However, further studies are necessary to establish risk factors for port site metastases and determine the role of chemotherapy as well as surgical intervention.

\section{References:}

1. Ziprin P, Ridgway PF, Peck DH, Darzi AW. The theories and realities of port-site metastases: a critical appraisal. J Am Coll Surg. 2002;195:395-408.

2. Stockdale AD, Pocock TJ. Abdominal wall metastasis following laparoscopy: A case report. Eur J Surg Oncol. 1985;11:373375

3. Pearlstone DB, Feig BW, Mansfield PF. Port site recurrences after laparoscopy for malignant disease. Semin Surg Oncol. 1999; 16:307-312.

4. Schaeff B, Paolucci V, Thomopoulos J. Port site recurrences after laparoscopic surgery: A review. Dig Surg. 1998;15:124-134.

5. Rangarajan M. Multiple port-site and subcutaneous metastases following palliative laparoscopic surgery for advanced gastric cancer. Ann Acad Med Singapore. 2007;36:875-876.
6. Sakurai K, Tanaka H, Lee T, et al. Port site metastasis after laparoscopic-assisted distal gastrectomy (LADG). Int Surg. 2013; 98:363-366.

7. Cava A, Román J, González Quintela A, Martín F, Aramburo P. Subcutaneous metastasis following laparoscopy in gastric adenocarcinoma. Eur J Surg Oncol. 1990;16:63-67.

8. Yasuda K, Adachi Y, Shiraishi N, Inomata M, Takeuchi H, Kitano S. Prognostic effect of lymph node micrometastasis in patients with histologically node-negative gastric cancer. Ann Surg Oncol. 2002;9:771-774.

9. Neuhaus SJ, Texler M, Hewett PJ, Watson DI. Port-site metastases following laparoscopic surgery. Br J Surg. 1998;85:735741.

10. Curet MJ. Port site metastases. Am J Surg. 2004;187:705-712.

11. Jones DB, Guo L, Reinhard Mk, et al. Impact of pneumoperitoneum on trocar site implantation of colon cancer in hamster model. Dis Colon Rectum. 1995;38:1182-1188.

12. Lee BR, Tan BJ, Smith AD. Laparoscopic port site metastases: incidence, risk factors, and potential preventive measures. Urology. 2005;65:639-644.

13. Martinez J, Targarona EM, Balagué C, Pera M, Trias M. Port site metastasis; An unresolved problem in laparoscopic surgery: a review. Int Surg. 1995;80:315-321.

14. Ouellette JR, Ko AS, Lefor AT. The physiologic effects of laparoscopy: Applications in oncology. Cancer. J 2005;11:2-9.

15. Deogracias ML, Rodríguez-Sanjuán JC, de la Torre F, et al. Absence of port-site metastases following staging laparoscopy for gastric carcinoma. Rev Esp Enferm Dig. 2006;98:755-759. 